\title{
Perturbative hydrodynamic Gross-Pitaevskii treatment for Bose-Einstein condensate in infinite length ring with disorder
}

\author{
D. B. Abdullaev ${ }^{1}$, B. Abdullaev${ }^{1}$, and M. M. Musakhanov \\ ${ }^{1}$ Institute of Applied Physics, National University of Uzbekistan, \\ Tashkent 100174, Uzbekistan \\ ${ }^{2}$ National University of Uzbekistan, Tashkent 100174, Uzbekistan \\ abdullaev_davron@mail.ru, bakhodir_abdullaev@yahoo.com, yousufmm@list.ru
}

PACS 47.37.+q, 67.10.Jn, 67.85.Jk

DOI 10.17586/2220-8054-2015-6-2-213-215

For the derivation of the dilute Bose-Einstein condensate density and its phase, we have developed the perturbative approach for the solution of the stationary state couple Gross-Pitaevskii hydrodynamic equations. The external disorder potential is considered as a small parameter in this approach. We have derived expressions for the total density, condensate density, condensate density depletion and superfluid velocity of the Bose-Einstein condensate in an infinite length ring with disorder potential having a general form. For the delta correlated disorder, the explicit analytical forms of these quantities (except the superfluid velocity) have been obtained.

Keywords: Bose-Einstein condensate in ring, Gross-Pitaevskii hydrodynamic equations, disorder potential, condensate density, condensate density depletion, delta correlated disorder.

Received: 2 February 2015

The Gross-Pitaevskii equation $[1,2]$ :

$$
i \hbar \frac{\partial \psi(\boldsymbol{x}, t)}{\partial t}=\left(-\frac{\hbar^{2}}{2 m} \nabla^{2}+U(\boldsymbol{x})+g|\psi(\boldsymbol{x}, t)|^{2}\right) \psi(\boldsymbol{x}, t) .
$$

is a powerful approach for the description of the Bose-Einstein condensate of the dilute ultracold atomic Bose gases [3], which have been recently observed in many experiments on cooling of atoms in magnetic traps and laser radiation (see references on experimental papers in [3]). In Eq. (1), the term proportional to g describes the contact interaction between two atoms in the s-scattering approximation.

With the existence of the external potential $U(\boldsymbol{x})$ (confining trap or disorder potential) and a fluidity flow, the condensate wave function $\psi(\boldsymbol{x}, t)=\sqrt{n(\boldsymbol{x}, t)} e^{i S(\boldsymbol{x}, t)}$ becomes a function not only of the condensate density $n(\boldsymbol{x}, t)$, but also of its phase $S(\boldsymbol{x}, t)$. For that case, it is rather convenient to describe a system by the couple hydrodynamic equations for the condensate density and its phase, originating from the Gross-Pitaevskii equation:

$$
\begin{gathered}
\frac{\partial n}{\partial t}+\nabla\left(n \boldsymbol{v}_{s}\right)=0 \\
m \frac{\partial \boldsymbol{v}_{s}}{\partial t}+\nabla\left(\frac{1}{2} m \boldsymbol{v}_{s}^{2}+U(\boldsymbol{x})+g n-\frac{\hbar^{2}}{2 m \sqrt{n}} \nabla^{2} \sqrt{n}\right)=0 .
\end{gathered}
$$

Here, $m$ is the mass of an atom, the superfluid velocity is expressed by formula $\boldsymbol{v}_{s}=\hbar \nabla S(\boldsymbol{x}, t) / m$ and for simplicity, we have omitted arguments in expressions for $n(\boldsymbol{x}, t)$, $S(\boldsymbol{x}, t), \boldsymbol{v}_{s}(\boldsymbol{x}, t)$. For the stationary case, when time derivatives of the condensate density and the superfluid velocity are equal to zero, Eq. (2) reduces to: 


$$
\begin{gathered}
\nabla\left(n \boldsymbol{v}_{s}\right)=0 \\
\nabla\left(\frac{1}{2} m \boldsymbol{v}_{s}^{2}+U(\boldsymbol{x})+g n-\frac{\hbar^{2}}{2 m \sqrt{n}} \nabla^{2} \sqrt{n}\right)=0 .
\end{gathered}
$$

Here and below, the kinetic energy of a superfluidity and external potential are expressed in $g n^{0}$ units and we introduce $y=\left(n / n^{0}\right)^{1 / 2}$. Condensate density $n^{0}$ is the solution of the second equation of Eqs. (3) obtained at $U(\boldsymbol{x})=0$.

We will find the solution of Eqs. (3) using the perturbative approach, substituting in them expansions: $y(\boldsymbol{x})=1+\varepsilon_{1}(\boldsymbol{x})+\varepsilon_{2}(\boldsymbol{x})$ and $\boldsymbol{v}_{s}(\boldsymbol{x})=\boldsymbol{v}_{s 0}+\boldsymbol{v}_{s 1}(\boldsymbol{x})+\boldsymbol{v}_{s 2}(\boldsymbol{x})$, where numerical indexes mean the order of the correction, the superfluid velocity $\boldsymbol{v}_{s 0}$ corresponds to case $U(\boldsymbol{x})=0$, and using in these expansions the Fourier integral transforms. For weak disorder, we consider $U(\boldsymbol{x})$ as the first order correction and also use its Fourier transform.

Our interest is in the calculation of the total density of particles and the condensate density of particles $\frac{\langle n(\boldsymbol{x})\rangle}{n^{0}}=\left\langle y^{2}(\boldsymbol{x})\right\rangle, \frac{\left\langle n_{0}(\boldsymbol{x})\right\rangle}{n^{0}}=\langle y(\boldsymbol{x})\rangle^{2}$, respectively, averaged over the ensemble of the disorder potential. We denote the averaging procedure by $\langle\cdots\rangle$. We point out the property $\langle U(\boldsymbol{x})\rangle=0$ for the disorder potential and existence of its correlator $\left\langle U(\boldsymbol{x}) U\left(\boldsymbol{x}^{\prime}\right)\right\rangle=$ $R\left(\left|\boldsymbol{x}-\boldsymbol{x}^{\prime}\right|\right)$, whose Fourier transform is $\left\langle U(\boldsymbol{k}) U\left(\boldsymbol{k}^{\prime}\right)\right\rangle=(2 \pi)^{1} \delta\left(\boldsymbol{k}+\boldsymbol{k}^{\prime}\right) R(\boldsymbol{k})$.

We obtain expressions for the total density, condensate density, and condensate density depletion, respectively:

$$
\begin{gathered}
\frac{\langle n(\boldsymbol{x})\rangle}{n^{0}}=1+\frac{1}{1-m \boldsymbol{v}_{s 0}^{2}} \int \frac{d k}{2 \pi} \frac{R(\boldsymbol{k})\left[\frac{\hbar^{2} \boldsymbol{k}^{2}}{2 m g n^{0}}-6 m \boldsymbol{v}_{s 0}^{2}\right]}{\left[\frac{\hbar^{2} \boldsymbol{k}^{2}}{2 m g n^{0}}+2\left(1-m \boldsymbol{v}_{s 0}^{2}\right)\right]^{2}}, \\
\frac{\left\langle n_{0}(\boldsymbol{x})\right\rangle}{n^{0}}=1+\frac{1}{1-m \boldsymbol{v}_{s 0}^{2}} \int \frac{d k}{2 \pi} \frac{R(\boldsymbol{k})\left[\frac{\hbar^{2} \boldsymbol{k}^{2}}{2 m g n^{0}}-1-5 m \boldsymbol{v}_{s 0}^{2}\right]}{\left[\frac{\hbar^{2} \boldsymbol{k}^{2}}{2 m g n^{0}}+2\left(1-m \boldsymbol{v}_{s 0}^{2}\right)\right]^{2}}, \\
\frac{\left\langle n(\boldsymbol{x})-n_{0}(\boldsymbol{x})\right\rangle}{n^{0}}=\int \frac{d k}{2 \pi} \frac{R(\boldsymbol{k})}{\left[\frac{\hbar^{2} \boldsymbol{k}^{2}}{2 m g n^{0}}+2\left(1-m \boldsymbol{v}_{s 0}^{2}\right)\right]^{2}}
\end{gathered}
$$

and the expression for the superfluid velocity:

$$
\left\langle\boldsymbol{v}_{s}(\boldsymbol{x})\right\rangle=\boldsymbol{v}_{s 0}-\frac{\boldsymbol{v}_{s 0}}{1-m \boldsymbol{v}_{s 0}^{2}} \int \frac{d k}{2 \pi} \frac{R(\boldsymbol{k})\left[\frac{\hbar^{2} \boldsymbol{k}^{2}}{2 m g n^{0}}-4-2 m \boldsymbol{v}_{s 0}^{2}\right]}{\left[\frac{\hbar^{2} \boldsymbol{k}^{2}}{2 m g n^{0}}+2\left(1-m \boldsymbol{v}_{s 0}^{2}\right)\right]^{2}} .
$$

We consider the Bose-Einstein condensate in the one dimensional ring trap, whose torus circumference is given by the length $L$. The trap of this geometry has a periodic boundary condition. Thus, all properties and quantities of the system must appear with periodic length $L$. For instance, the delta correlated disorder potential correlation function is:

$$
R(\boldsymbol{x})=r \sum_{j=-\infty}^{j=\infty} \delta(\boldsymbol{x}-L j)
$$

where $r=U_{0}^{2}$ for the average strength $U_{0}$ of the disorder potential. According to this, they must be expanded to the Fourier series with discrete values for the wave vectors. However, for a limit of the infinite length $L \rightarrow \infty$, these series on discrete wave values can easily be 
transformed into the integral expressions on the continuous wave vector for quantities, in which we have interest, by replacing $\sum \rightarrow \int L d k / 2 \pi$.

For $L \rightarrow \infty$, the asymptotic expressions for the ring geometry total density, condensate density, and condensate density depletion, respectively, are:

$$
\begin{gathered}
\frac{\langle n(\boldsymbol{x})\rangle}{n^{0}}=1+\frac{\pi p^{1 / 2} r\left(1-4 m \boldsymbol{v}_{s 0}^{2}\right)}{2^{3 / 2} L\left(g n^{0}\right)^{2}\left(1-m \boldsymbol{v}_{s 0}^{2}\right)^{5 / 2}}, \\
\frac{\left\langle n_{0}(\boldsymbol{x})\right\rangle}{n^{0}}=1+\frac{\pi p^{1 / 2} r\left(1-7 m \boldsymbol{v}_{s 0}^{2}\right)}{2^{5 / 2} L\left(g n^{0}\right)^{2}\left(1-m \boldsymbol{v}_{s 0}^{2}\right)^{5 / 2}}, \\
\frac{\left\langle n(\boldsymbol{x})-n_{0}(\boldsymbol{x})\right\rangle}{n^{0}}=\frac{\pi p^{1 / 2} r}{2^{5 / 2} L\left(g n^{0}\right)^{2}\left(1-m \boldsymbol{v}_{s 0}^{2}\right)^{3 / 2}},
\end{gathered}
$$

where $p=2 m L^{2} g n^{0} /\left((2 \pi)^{2} \hbar^{2}\right)$.

In conclusion, we have developed the perturbative approach for the stationary state hydrodynamic Gross-Pitaevskii equations in the external disorder potential and applied it to investigate the one dimensional Bose-Einstein condensate of ring geometry. We have found expressions for the total density, condensate density, condensate density depletion and superfluid velocity of that condensate for the disorder potential, which has a general form, considering this potential perturbatively. For the delta correlated disorder, the explicit analytical forms of these quantities (except the superfluid velocity) have been obtained. It is interesting that for the superfluid velocity $\boldsymbol{v}_{s 0}=0$, i.e. no initial superfluid flow in the condensate, above expressions for the total density, condensate density, and condensate density depletion reproduce the same expressions for the static Bose-Einstein condensate [4].

\section{References}

[1] E. M. Lifshitz and L. P. Pitaevskii. Statistical Physics, Part 2: Theory of Condensed State, Course of Theoretical Physics, V. 9, Elsevier Ltd., Amsterdam 1980, 387 p.

[2] L. Pitaevskii and S. Stringari, Bose-Einstein Condensation, Oxford University Press, Oxford, 2003,492 p.

[3] L. Dalfovo, S. Giorgini, L. P. Pitaevskii, and S. Stringari. Theory of Bose-Einstein condensation in trapped gases. Rev. Mod. Phys., 71, P. 463 -512 (1999).

[4] C. Krumnow and A. Pelster. Dipolar Bose-Einstein condensates with weak disorder. Phys. Rev. A, 84, P. 021608(R) (2011). 